\title{
Diagnostic informativity of the volume MDCT-angiography and MR-cholangiography in the pre- and intraoperative periods for the examination of donors of a liver fragment
}

\author{
Feruz Gafurovich NAZIROV, Nigora Muxsumovna DJURAEVA, Nargiza Tulkinovna VAKHIDOVA, Oybek \\ Avazxanovich OMONOV and Umid Ravshanovich SALIMOV \\ Republican Specialized Scientific and Practical Medical Center of Surgery named after academician V.Vakhidov Tashkent, Uzbekistan \\ Corresponding author's Email: sidikovan@yandex.ru
}

\section{ABSTRACT}

Introduction. The first transplantation of a liver fragment from a living donor was performed in Uzbekistan on February 12, 2018 at the Republican Specialized Scientific Practical Medical Center of Surgery named after acad.V. Vakhidov. This event laid the foundation for a new direction for domestic clinical practice that meets the current level of world medicine development. Aim. The aim of the study was to determine the diagnostic information content of preoperative data of the volume multi detector computed tomography (MDCT) angiography and magnetic resonance cholangiography (MRCG) when compared with intraoperative ones at examining related donors for liver fragment transplantation (LFT). Methods. Total of 88 potential donors of a liver fragment aged from 19 to 58 years ( 53 men and 35 women) were examined for the period 2017-2019. Sixteen donors were undergone liver resection to obtain a transplant: the right lobe of the livers in 12 people and the left lobe in 4 people. Results. Compared with intraoperative data, the main arteries supplying the transplant planned for resection were identified with MDCT-angiography in 98.4\% of cases $(\mathrm{P}<0.05)$. Variations of the portal bed according to MDCT-angiography in comparison with intraoperative ones were determined in $93.8 \%$ of cases $(P<0.05)$. Intraoperatively revealed the main trunks of the venous outflow were determined by MDCT-angiography in $95.7 \%$ of cases $(\mathrm{P}<0.05)$. Recommendation. We suggest that MDCT angiography and MRCG is a highly informative and important method for estimation the condition of the liver in transplant planning.

Original Article

PII: S225199391900024-9

Rec. 11 October 2019

Rev. 20 November 2019

Pub. 25 November 2019

\section{Keywords}

Lung Pathology,

Video-Assisted

Thoracoscopy,

Geprotsel,

Hemostasis,

Aerostasis

\section{INTRODUCTION}

The undoubted advantage of receiving a transplant from a living donor in the planned preparation of related donors and the possibility of a qualitative choice of donor hepatic parenchyma is an important factor for a favorable prognosis of the surgery's outcome. Negative hemodynamic and drug effects are practically excluded for the donor in the case of a related transplantation at the preoperative stage [1-4]. The indisputable advantage of a liver fragment transplantation (LFT) from a living related donor is independence from the system of providing cadaveric organs, which means that planning the timing of the operation depending on the state of the recipient [5-10].

In contrast to cadaveric liver transplantation, the use of an organ fragment from a close relative allows to count on its more favorable immunological adaptation in the recipient's body [11-13]. The first transplantation of a liver fragment from a living related donor was performed in Uzbekistan on February 12, 2018 at the Republican Specialized Scientific Practical Medical Center of Surgery (RSSPMCS) named after acad. V.Vakhidov. This event laid the foundation for a new direction for domestic clinical practice that meets the current level of world medicine development [12, 14].

All main disadvantages of related liver transplantation are associated with a potential risk to the health and life of the donor which causes certain skepticism and ethical questions among the general medical community. Thereby, one of the important aspects of planning the surgery of related donors is a detailed examination of the selected potential donor [11, 15-20]. After conducting clinical and laboratory studies instrumental examination methods are performed at the final stage, which includes volume MDCT angiography and magnetic resonance cholangiography (MRCG). The main task is to clarify the technical capabilities of obtaining a full-fledged liver transplant while fully preserving the possibilities of donor rehabilitation. It is necessary to determine the following for doing this: 1) the size and weight of the donor liver fragment to be removed and the remaining part (liver stump); 2) an anatomical version of the circulatory system structure 
entering the porta of the donor liver, venous outflow from the organ and architectonics of the biliary system [13, 21-24]. Since its introduction to the clinics, MDCT angiography has become the main method of instrumental examination of related donors of a liver fragment $[2,6,16,25,26]$. In accordance with the requirements of transplant doctors, a special MDCT scan protocol (all in one) was developed, which includes four phases of the study: native, arterial, portal and venous [27-31]. To determine the diagnostic information content of the preoperative results of volume MDCT angiography and MRCG in the rebound of potential liver donors, as well as their comparison with the intraoperative anatomical picture.

\section{MATERIAL AND METHODS}

\section{Ethical approval}

The review board and ethics committee of RSCS named after acad. V.Vakhidov approved the study protocol and informed consents were taken from all the participants.

Total of 88 potential donors of a liver fragment - 53 men and 35 women - aged from 19 to 58 years (the mean age was $34.1 \pm 9.06$ years) were examined. All patients were examined in RSSPMCS named after acad.V. Vakhidov for the period 2017-2019. At the time of the present study, 16 donors were undergone liver resection to obtain a transplant: in 12 people (the right lobe, RL), in 4 people (the left lobe of the liver, LL). All related donors underwent four-phase MDCT angiography and MRCG of liver. The investigations were carried out on the wide-detector MDCT (Aquilion One - 640 Genesis, Cannon, Japan), as well as MRI on the Signa HDxt with a magnetic field of $1.5 \mathrm{~T}(\mathrm{GE})$. The rate of contrast agent (CA) introduction was $5.0-5.5 \mathrm{ml} / \mathrm{sec}$, the amount of CA varied $100 \pm 10 \mathrm{ml}$ (Unigexol 350).

MDCT investigation began with an inserting of the catheter into the external cubital vein followed by the placement and instruction of the patient about the progress of the upcoming procedure. An automatic syringeinjector of the "Ulrich" company was used for injection, having previously collected 100-120 $\mathrm{ml}$ of an iodinecontaining contrast preparation into the flask for injection with an iodine concentration of $350-370 \mathrm{mg} / \mathrm{ml}$ and an injection rate of 4.5-5.0 ml/s. The selection protocol in this case was a multiphase scanning protocol with selected parameters, which included a native study, arterial, portal and delayed phases (phase of the hepatic veins) scans performed on one breath hold in each phase (Table 1). Magnetic resonance imaging (MRI) investigation of the patient began with laying on his back using a surface receiving coil for the body. The following programs are used for the bile ducts investigation. A) Localizer (sighting image); B) T2-COR 2D FIESTA FATSAT (breath holding) - for more precise setting of slices for the MRCG program; C) 3D MRCG program without holding one's breath, but using the 3D MRCG Rtr ASSET navigator-the program is synchronized with the movement of the diaphragm. The main parameters of the MRCG programs are shown in Table 2.

Table 1 - Main parameters of the MDCT scanning programs

\begin{tabular}{lccc}
\hline Parameters & Arterial phase & Portal phase & $\begin{array}{c}\text { Delayed phase } \\
\text { (phase of the hepatic veins) } \\
\text { Liver hilum }\end{array}$ \\
\hline Level & Liver hilum & Liver hilum & $50-60$ sec \\
\hline $\begin{array}{l}\text { Scan delay: Duration of study phases, } \\
\text { automatic bolus tracking }\end{array}$ & $15-20$ sec $120+\mathrm{HU}$ & $30-40 \mathrm{sec}$ & 120 \\
\hline Tube voltage $(\mathrm{kV})$ & 120 & 120 & 140 \\
\hline Effective mas/ Quality ref mas & 140 & 140 & $0.5 \mathrm{~s}$ \\
\hline Rotation time & $0.5 \mathrm{~s}$ & $0.5 \mathrm{~s}$ & \\
\hline
\end{tabular}

Table 2. The main parameters of the MRCG programs

\begin{tabular}{lcc}
\hline Parameters & COR 2D FIESTA FATSAT & 3D MRCG Rtr ASSET \\
\hline Layer & 1 & 1 \\
\hline Quantity of slices & $15-20$ & $40-50$ \\
Diet factor & 0 & $50 \%$ \\
\hline FOV & $300-330 \mathrm{~mm}$ & $380 \mathrm{~mm}$ \\
\hline FOV phase & $100 \%$ & $100 \%$ \\
\hline Slice thickness & $3-4.5 \mathrm{~mm}$ & $1.5 \mathrm{~mm}$ \\
\hline TR & $1240 \mathrm{~m} / \mathrm{s}$ & $1800 \mathrm{~m} / \mathrm{s}$ \\
\hline TE & $87 \mathrm{~m} / \mathrm{s}$ & $681 \mathrm{~m} / \mathrm{s}$ \\
\hline Averaging & 1 & 1 \\
\hline Flip angel & $150 \mathrm{deg}$. & $170 \mathrm{deg}$. \\
\hline Fat sat (with fat suppressed) & + & - \\
\hline
\end{tabular}


Up to and during conducting this research, 16 (18.4\%) of the 88 potential examined donors have been operated on to collect a liver fragment. In RSSPMCS named after acad.V. Vakhidov, 4 liver transplants were performed, and the rest were operated on at Yashoda Hospital (India). Depending on the anthropometric data of the recipient, the volume of the transplant and the resected share of the donor were determined. Resection of the right lobe of the liver in order to obtain a transplant from the right lobe was performed in 12 (75\%) donors and in $4(25 \%)$ the left lobe of the liver was used as a transplant. The vascular and biliary anatomy of the liver and the mass of the resected liver fragment were also examined during the surgery.

During the surgery performed in donors it was revealed that in 8 (66.6\%) from 12 who were resected the right lobe of the liver, the source of arterial circulation was own hepatic artery (OHA) extending from the celiac trunk, which corresponded to type I according to MDCT angiography and was identified in 7 (58.3\%) donors at the preoperative stage. In 3 donors (25\%) we revealed a discharge of the right hepatic artery from the common hepatic one which corresponds to type II at MDCT and was detected in 4 (33.3\%) donorsr (8.3\%) we registered a discharge of the right hepatic artery (RHA) from the superior mesenteric artery (SMA), which corresponded to type IV at MDCT.

In 4 donors the left lobe of the liver was used as a transplant. Intraoperatively, it was revealed that in 3 (75\%) donors the left lobe was supplied from the OHA basin, which corresponded to type I according to MDCT and was detected in 3 (75\%) donors. There was an abnormality of the left hepatic artery (LPA) from the total hepatic artery (OPA) in 1 donor (25\%) which corresponds to type II according to MDCT and completely coincided with preoperative data. In all operated 16 donors blood supply of segment IV of the liver was also evaluated intraoperatively. Moreover, in $10(62.5 \pm 5.2 \%)$ of them blood supply was carried out due to the left lobar hepatic artery. Blood supply of the segment IV from the right hepatic artery was detected in $4(25 \pm 4.1 \%)$ donors and in 2 $(12.5 \pm 3.5 \%)$ a mixed type was revealed (left and right hepatic arteries). All donors were performed 3D reconstruction of the arterial bed of the liver (Figure 1). When evaluating the portal vein in 14 (87.5 $\pm 3.6 \%)$ of the 16 operated donors, type I of portal vein (PV) branching (classical) was revealed. Trifurcation of the portal vein was detected in 1 donor $(6.25 \pm 2.4 \%)$ and in 1 donor at MDCT it was described as trifurcation, but type I was intraoperatively detected due to the close separation of the branch of the liver segment VI from bifurcation. 3D models of the liver portal bed were also reconstructed (Figure 2).

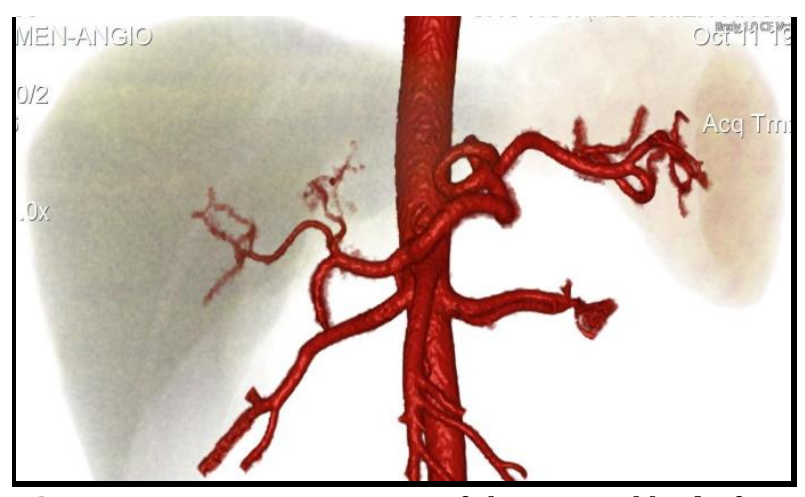

Figure 1. 3D-reconstruction of the arterial bed of the liver and abdominal aorta

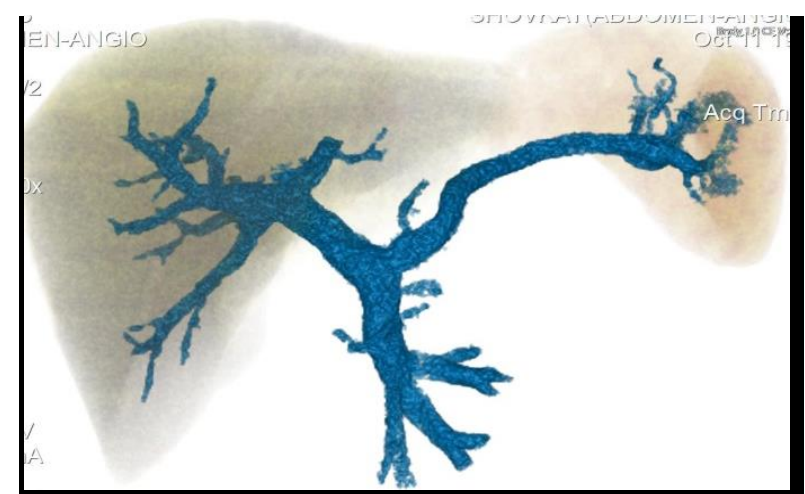

Figure 2. 3D-model of the of the liver portal bed

An intraoperative assessment of venous outflow from the liver revealed that type I was detected in 9 (56.3\%) patients - this is a separate inflow of the right hepatic vein (RHV), the median hepatic vein (MHV) and the left hepatic vein (LHV) into the postcava. In 4 cases (25\%), type II of PV branching was observed, it was when MHV and LHV fell into a single mouth and type III, fusion of MHV and LHV into a single trunk was observed in 3 (18.7\%) donors. In 12 donors who performed right-sided liver resection, RHV fell into postcava as a single trunk. Along with RHV in 2 donors (12.5\%), an additional right vein from the segment VIII of the liver independently flowing into postcava was revealed. In 1 donor (6.3\%), an additional lower right hepatic vein from the segment V to MHV was noted, which was reflected in the MDCT protocol. In 7 donors (43.7\%), small additional lower right hepatic veins flowing into postcava were revealed intraoperatively. These small branches were hemodynamically insignificant and were ligated during the resection. 3D models of venous outflow of the liver were also used for volumetry of the liver (Figure 3). 


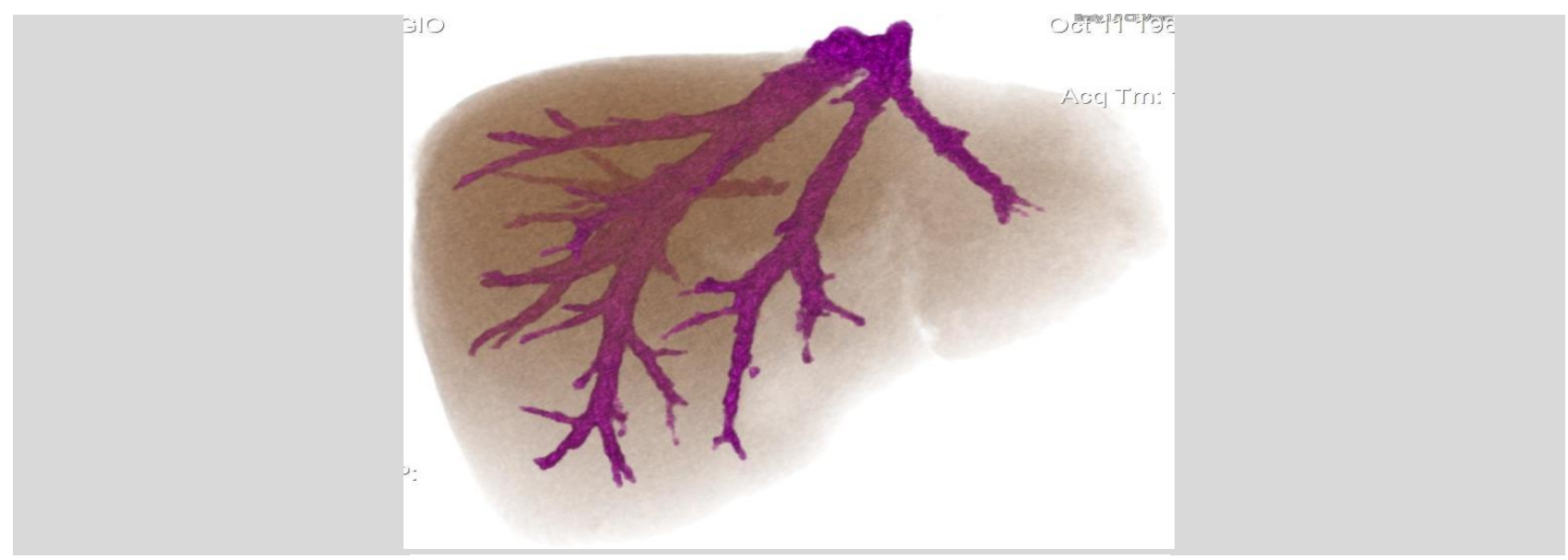

Figure 3. 3D-reconstruction of the liver venous outflow

\section{The transplant volume}

When weighing during the operation the obtained transplant, the mass of the right lobe ranged from 5601420 grams, the mass of the left lobe - from 240 to 670 grams. When calculating the volume of the transplant, absolute coincidence with intraoperative data was observed in 3 donors (18.7\%) of the right lobe and in 1 donor (6.2\%) of the left lobe of the liver. For the purpose of additional analysis of the obtained data, axial images obtained during MDCT angiography in the Digital Imaging and Communications in Medicine (DICOM) format were processed using the Vitrea (version 7.4.0.462, Vital Images) software, which allowed us to build virtual objects of the zones of interest to us and to study, based on 3D maps, the relative position of the vascular structures of the liver, as well as visually assess the future fragment of the liver and calculate the volume of the future transplant. Examples of reconstructed 3D maps are shown in Figures 4 and 5.

Comparative data of MRCG with intraoperative ones were as follows. It was revealed that diagnosed type I on MRCG was detected in 13 (82\%) from 16 operated patients. In 1 donor (6.2\%), type I was determined on MRCG before the surgery, but type II was detected during surgery, which was explained by the close parallel lining of two right segmental ducts. In 1 donor (6.2\%), 3 mouths of the bile ducts were revealed which corresponded to type III and was explained by the close fusion of two branches of the right anterior bile ducts to confluence. And in 1 (6.2\%) donor we revealed 2 mouths of the transplant of the liver left lobe. Diagnostic informativity and accuracy of MDCT angiography in determining options and types of arterial and portal blood supply to the liver, as well as venous outflow pathways are presented in Table $3(\mathrm{P}<0.05)$. Comparison of MDCT angiography and intraoperative data showed that the average error for the right lobe was $100 \pm 21.8$ grams, for the left lobe 42.2 \pm 5.3 grams ( $\mathrm{P}<0.05$; Table 4). Diagnostic informativity and accuracy of MR cholangiography in determining biliary ducts are presented in Table $5(\mathrm{P}<0.05)$.

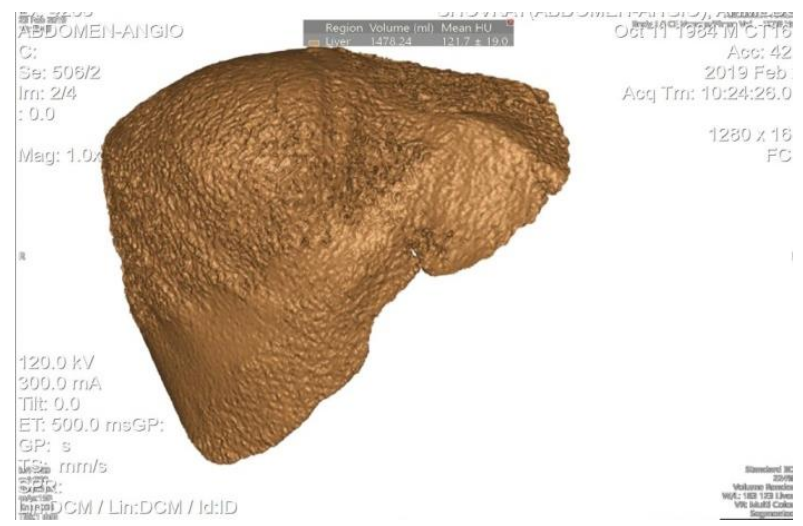

Figure 4. 3D-model of the liver in the frontal projection

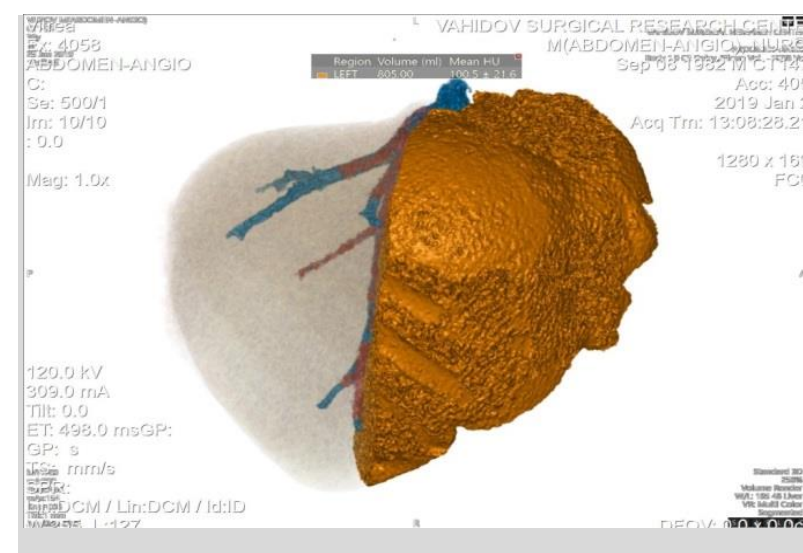

Figure 5. A 3D-model of the left lobe of the liver

Table 3. Diagnostic informativity of MDCT angiography in comparison with intraoperative data

\begin{tabular}{lccc}
\hline Rates of the method informativity & $\begin{array}{c}\text { Arterial blood supply } \\
\text { of the liver }\end{array}$ & $\begin{array}{c}\text { Variant of portal } \\
\text { blood supply to the }\end{array}$ & $\begin{array}{c}\text { Main ways of venous } \\
\text { outflow }\end{array}$ \\
\hline Sensitivity \% & 96.8 & 97.1 & 95.9 \\
\hline Accuracy \% & 92.5 & 93.7 & 91.3 \\
\hline
\end{tabular}


Table 4. The average mass of the liver fragment (transplant) obtained on MDCT angiography and during surgery

\begin{tabular}{lcc}
\hline Liver fragments & $\begin{array}{c}\text { The average mass of the alleged } \\
\text { transplant according to MDCT, grams }\end{array}$ & $\begin{array}{c}\text { The average mass of the liver fragment } \\
\text { obtained intraoperatively, grams }\end{array}$ \\
\hline Right lobe & $811.7 \pm 30.5$ & $788.8 \pm 27.2$ \\
\hline Left lobe & $327.5 \pm 31.3$ & $369 \pm 37.8$ \\
\hline
\end{tabular}

Table 5. Diagnostic informativity of MR cholangiography vs. intraoperative data

\begin{tabular}{lcc}
\hline Rates of method informativity & Right lobar duct & Left lobar duct \\
\hline Sensitivity \% & 96.8 & 97.1 \\
\hline Accuracy \% & 92.5 & 93.3 \\
\hline
\end{tabular}

\section{CONCLUSION}

According to preoperative MDCT angiography, the most common variant anatomy of the arterial blood supply to the liver was type Michels [31] classification, type I portal blood circulation according to Nakamura et al. [31] and type I venous outflow from the liver according to Soyer [32], which were determined intraoperatively at 95.7 \% cases $(\mathrm{P}<0.05)$. MDCT angiography and MRCG in the definition of variant anatomy were $97.8 \%$ and $96.8 \%$, respectively. The volume (mass) of the planned liver transplant obtained with MDCT volumetry was confirmed with intraoperative data in $92.8 \%(\mathrm{P}<0.05)$. The data obtained indicate the high information content and the importance of these methods in planning liver transplantation.

\section{DECLARATIONS}

\section{Acknowledgements}

This work was supported by Republican Specialized Scientific and Practical Medical Center of Surgery named after academician V.Vakhidov, Tashkent, Uzbekistan

\section{Authors' contributions}

All authors contributed equally to this work.

\section{Competing interests}

The authors declare that they have no competing interests.

\section{REFERENCES}

1. Gauthier CB, Konstantinov BA, Tsirulnikova OM Liver Transplantation: A Guide for Physicians. 2008; 15-91.

2. Gauthier $\mathrm{CB}$, Tsirulnikova $\mathrm{OM}$, Filin $\mathrm{AB}$ et al. Experience of 25 transplantations of the right lobe of the liver from a living related donor. Annals of the Russian Scientific Center of Medical Sciences. 2002; (11): 30-36.

3. Kim E.F. Clinical and surgical aspects of intravital donation of liver fragments. Abstract. Dis. Dr. med. Science. 2008: 15131.

4. Caruso S, Miraglia R, Maruzzelli L, Gruttadauria S, Luca A, Gridelli B. Imaging in liver transplantation. World J Gastroenterol 2009; (15): 675-83. Google Scholar ; https://doi.org/10.3748/wjg.15.675

5. Kitaev VM, Belova IB, Kitaev CB. Contrast agent for CT. 2008. Computed tomography for liver diseases. 1 (1.3): 17.

6. El-Badrawy A, El-Nahas M, El-Hendawy A. Preoperative evaluation of living donor for liver transplantation using 64 MDCT. Journal of Medicine \& Biomedical Sciences. 2010 Aug; 1(3). Google Scholar

7. Gautier SV, Tsiroulnikova OM, Moysyuk YG, Akhaladze DG, Tsiroulnikova IE, Silina OV, Khizroev KM, Monakhov AR, Chekletsova EV, Pets VA, Poptsov VN. Liver transplantation in children: six-year experience analysis. Russian Journal of Transplantology and Artificial Organs. 2014 Sep 24; 16(3):54-62. Google Scholar ; https://doi.org/10.15825/1995-11912014-3-54-62

8. Kodama Y, Ng CS, Wu TT, Ayers GD, Curley SA, Abdalla EK, et al. Comparison of CT methods for determining the fat content of the liver. AJR Am J. Roentgenol. 2007; 188:1307. Google Scholar ; https://doi.org/10.2214/AJR.06.0992

9. Ma X, Holalkere NS, Kambadakone RA, Mino-Kenudson M, Hahn PF, Sahani DV. 2009. Imaging-based quantification of hepatic fat: Methods and clinical applications. Radiographics, 29 (1253): 77. Google Scholar; https://doi.org/10.1148/rg.295085186

10. Strasberg SM, Belghiti J, Clavien PA, Gadzijev E, Garden JO, Lau WY, et al. The Brisbane 2000 terminology of liver anatomy and resection. Terminology committee of the International Hepato-Pancreatico-Biliary Association. HPB. 2000; 2 (333):9. Google Scholar ; https://doi.org/10.1016/S1365-182X(17)30755-4 
11. Balakhnin PV, Tarazov PG, Polikarpov PA, et al. Variants of arterial anatomy of the liver according to 1511 angiographies. Ann. chir. Hepatol. 2004; 9 (2): 14-21. Google Scholar ;

12. Nazirov FG, Akbarov MM, Saydazimov EM, Nishanov MSh, Khakimov YuU. The use of modern technology for anatomical resections of the liver. Bulletin of the Association of Physicians of Uzbekistan. 2014; 4: 28-31.

13. Couinaud C. Liver anatomy: portal or biliary segmentation. Paris, 1957: 320. Google Scholar

14. Nazirov FG, Akbarov MM, Nishanov MSh, Saydazimov EM, Khakimov YuU. Ways to improve the results of extensive anatomical liver resections. Congress material "Actual problems of surgical hepatology". Donetsk: 2013; 37-38.

15. Egorov VI, Iashina NI, Karmazanovsky GG, Fedorov AV. CT-angiography as a reliable method for delineation of celiac and mesenteric arteries lesions, variants and postoperative changes. Medical Visualization. 2009; 3:82-94. Google Scholar

16. Semenkov AV. Related donors for liver transplantation: selection, examination, surgical tactics. /Abstract. of PhD dis.M., 2003 - p. 21.

17. Catalano OA, Singh AH, Uppot RN, Hahn PF, Ferrone CR, Sahani DV. Vascular and biliary variants in the liver: Implications for liver surgery. Radiographics 2008; 28 (359): 78. Google Scholar ; https://doi.org/10.1148/rg.282075099

18. Li KW, Wen TF, Yan LN, Li B, Zeng Y, Zhao JC, Wang WT, Xu MQ, Yang JY, Ma YK, Chen ZY. Donor right hepatectomy in living donor liver transplantation: report of 143 cases. Hepato-gastroenterology. 2010 Sep 1;57(102-103):1232-6. Google $\underline{\text { Scholar }}$

19. Tanaka K, Yamada T. Living donor liver transplantation in Japan and Kyoto University: What can we learn? J Hepatol 2005; 42: 25-8. Google Scholar; https://doi.org/10.1016/j.jhep.2004.11.004

20. Zhuang ZG, Qian LJ, Gong HX, Zhou Y, Chai WM, Li QG, et al. Multidetector computed tomography angiography in the evaluation of potential living donors for liver transplantation: Single-center experience in China. Transplant Proc 2008; 40:2466-77. Prime PubMed (Link) ; https://doi.org/10.1016/j.transproceed.2008.08.031

21. Sandrikov VA, Semenkov AV, Khovrin VV, Kim SYu, Burmistrov DS, Korotneva NA, Bogdanov- Berezovsky AA. Possibilities of MR-cholangiography in the preoperative assessment of the variant structure of the bile ducts of liver fragments in living donors. Annals of Surgical Hepatology. (4): 57.

22. Kim KW, Lee J, Lee $\mathrm{H}$, Jeong WK, Won HJ, Shin YM, et al. Right lobe estimated blood-free weight for liver transplantation: Accuracy of automated blood-free CT volumetry--preliminary results. Radiology. 2010; 256:43340. Google Scholar ; https://doi.org/10.1148/radiol.10091897

23. Radtke A, Sotiropoulos GC, Nadalin S, Molmenti EP, Schroeder T, Saner FH, Sgourakis G, Cicinnati VR, Valentin-Gamazo C, Broelsch CE, Malago M. Preoperative volume prediction in adult live donor liver transplantation: 3-D CT volumetry approach to prevent miscalculations. Eur J Med Res. 2008 Jul 28; 13(7):319-26.. Google Scholar

24. Wang F, Pan KT, Chu SY, Chan KM, Chou HS, Wu TJ, et al. Preoperative estimation of the liver graft weight in adult right lobe living donor liver transplantation using maximal portal vein diameters. Liver Transpl 2011; 17:373-80. Lindenbraten L.D. Computed tomography Medical Radiology 2006; 92-98. Google Scholar ; https://doi.org/10.1002/lt.22274

25. Lee SW, Park SH, Kim KW, Choi EK, Shin YM, Kim PN, et al. Unenhanced CT for assessment of macrovesicular hepatic steatosis in living liver donors: Comparison of visual grading with liver attenuation index. Radiology 2007;244:479-85. Google Scholar ; https://doi.org/10.1148/radiol.2442061177

26. Krotova OA, Granov DA, Polysalov VN, Pirtskhalava TL, Borovik VV, Rutkin IO, Generalov MI, Maistrenko DN. Planning of surgical interventions on the liver based on the results of multilayer spiral computed tomography. Annals of Surgical Hepatology. 2010; 15 (2): 31-37.

27. Galyan TN, Khovrin VV, Kim SY, Kim EF, Semenkov AV. Assessment of variant vascular anatomy in potential living donors of a liver fragment according to magnetic resonance and multispiral computed tomography. Medical imaging. 2010; 5:37-43. Google Scholar

28. Lim JS, Kim MJ, Myoung S, Park MS, Choi JY, Choi JS, Kim SI. MR cholangiography for evaluation of hilar branching anatomy in transplantation of the right hepatic lobe from a living donor. American Journal of Roentgenology. 2008 Aug;191(2):537-45.. Google Scholar ; https://doi.org/10.2214/AJR.07.3162

29. Limanond P, Raman SS, Lassman C, Sayre J, Ghobrial RM, Busuttil RW, et al. Macrovesicular hepatic steatosis in living related liver donors: Correlation between CT and histologic findings. Radiology 2004; 230:276-80. Google Scholar ; https://doi.org/10.1148/radiol.2301021176

30. Michels NA. Newer anatomy of the liver and its variant blood supply and collateral circulation. Am J Surg. 1966; 112(3):337-347. DOI: https://dx.doi.org/10.1016\%2Fooo2-9610(66)90201-7 [PubMed] [CrossRef] [Google Scholar]

31. Nakamura T, Tanaka K, Kiuchi T, Kashara M, Oike F, Ueda M, et al. Anatomical variants and surgical strategies in right lobe liver donor liver transplantation: Lessons from 120 cases. Transplantation 2002; 73:1896-903. Google Scholar; https://doi.org/10.1097/00007890-200206270-00008

32. Soyer $P$, Levesque $M$, Legmann $P$, Fajadet $P$. Magnetic resonance imaging of malignant hepatic tumors in adults. $\underline{J}$ Radiol. 1992; 73(4):219-27. (PMID:1322459) [Article in French]. 